\title{
Article \\ Identification of the planetary magnetosphere boundaries with the wavelet multi-resolution analysis
}

\author{
Mauricio José Alves Bolzan 1,*(D), Ezequiel Echer 2, (D), Adriane Marques de Souza Franco 2,() and Rajkumar Hajra \\ 3, (D) \\ 1 Federal University of Jatai, Jatai, Brazil \\ Instituto Nacional de Pesquisas Espaciais (INPE), São José dos Campos, Brazil \\ 3 Indian Institute of Technology Indore, Simrol, Indore 453552, India \\ * Correspondence: mauricio-bolzam@ufj.edu.br
}

\begin{abstract}
The Haar wavelet decomposition technique is used to detect the planetary magnetosphere boundaries and discontinuities. We use the magnetometer data from the CASSINI and MESSENGER spacecraft to identify the abrupt changes in the magnetic field when the spacecraft crossed the magnetospheric bow shocks and magnetopauses of Saturn and Mercury, respectively. The results confirm that the Haar transform can efficiently identify the planetary magnetosphere boundaries characterized by the abrupt magnetic field changes. It is suggested that this technique can be applied to detect the planetary boundaries as well as the discontinuities such as the shock waves in the interplanetary space.
\end{abstract}

Keywords: Planetary magnetosphere; planetary bow shocks; planetary magnetopauses; Haar wavelet; Solar wind; Wavelet analysis

\section{Introduction}

Planetary magnetospheres are created owing to the interaction between the solar wind and the planetary body magnetic field and plasma environment. When a planet has an intrinsic magnetic field, its interaction with the impinging solar wind creates an "intrinsic" magnetosphere. For a non-magnetic planet, the solar wind is deflected by the ionized atmosphere (ionosphere) and a magnetosphere is "induced" due to the generation of currents in its atmosphere/ionosphere by the solar wind magnetized plasma flow [1].

As the planetary magnetospheres in the heliosphere are embedded in the supermagnetosonic, magnetized plasma (solar wind) that continuously escapes from the Sun's atmosphere, a stand bow shock (BS) is formed ahead of them. Following the BS, the solar wind plasma is shocked, decelerated, heated and deflected in a region called magnetosheath. This region extends from the shock to the magnetopause (MP), the latter being the outer boundary of the planetary magnetosphere where the planetary magnetic field and plasma dominate [2,3]. The magnetospheric shape, size and the positions of the boundaries (BS and MP) depend on the solar wind and internal (magnetosphere) conditions and can be highly variable depending on solar wind variations [3].

The magnetospheric size (or the MP location) is mainly determined by the planetary magnetic field intensity and the solar wind density in the planet orbit [4]. As the solar wind density at Mercury's orbit is very high (Mercury being the nearest planet to the Sun, at $\sim 0.4$ astronomical unit (au) distance) and the magnetic dipole of the planet is weak, the magnetosphere of Mercury is very small and its magnetopause is generally found about $2 R_{m e}$ (Mercury radii, $\sim 2438 \mathrm{~km}$ ) upstream the planet [5]. The solar wind Mach number $\left(M_{m s}\right.$, the ratio of the solar wind speed to the local magnetosonic speed) at Mercury is very low ( $\sim 2$ to 5 , for comparison, $M_{m s}$ near Earth varies between $\sim 5$ and $15)$, and consequently the properties of BS and magnetosheath are very susceptible to the variations in the upstream solar wind [6]. 
The Saturn's magnetosphere (at $\sim 9.5$ au from the Sun) is a complex system that has components from multiple physical sources [7]. The magnetosphere is fast rotating and has a strong internal source of material from the moons deep within the planetary system. Furthermore, the planet is embedded in the solar wind, and there are momentum, energy and mass exchanges with the solar wind [8]. Internal plasma sources of the Kronian magnetosphere include the rings, icy satellites and Titan and Saturn's upper atmosphere and ionosphere. Recently, Jackman et al. [9] analyzed 13 years of the CASSINI measurements in order to study the Saturn's BS and MP boundaries. They indicated the difficulty to determine the BS and MP under a range of solar wind driving conditions. This is suggested to be significantly contributed by the moon Enceladus which acts as a significant internal source of plasma [10].

In the preset work, we present a wavelet technique to identify the abrupt changes in the magnetic field at the magnetospheric boundary crossings. This technique decomposes the magnetic field data in frequency levels using the multi-resolution analysis (MRA). For this purpose, we analyzed the magnetic field measurements by CASSINI during its Saturn orbit insertion in 2004, and by MESSENGER during its first Mercury flyby in 2008. Both Saturn and Mercury are planets with intrinsic magnetospheres. The main aim of this work is to develop and demonstrate a technique to efficiently identify the planetary (magnetospheric) boundaries and discontinuities in the redspace plasmas, which can be used as an important tool in space research.

\section{Database and Method of Analysis}

In order to identify the magnetospheric BS and MP boundaries of Mercury and Saturn, we used two conjugate approaches, namely, the wavelet analysis to decompose the magnetic field vector component time series in dyadic scales, and the variance by scale analysis to identify the scales with the maximum energy. The variance by scale is an important approach to identify the sharp/abrupt changes in the magnetic field, and can be used to remove the long-term periodicities of the time series [see 11]. Bolzan et al. [11] used the Daubechies wavelet as this is more efficient in removing scales compared to, for example, the Haar wavelet [12]. However, the Haar wavelet is able to better identify the sharp changes in the time series compared to the Daubechies function. Thus, Haar wavelet decomposition technique will be used in the present work.

\subsection{Wavelet analysis}

Similar to the Fourier analysis, which decomposes a signal in sine wave components of different frequencies, the wavelet transform decomposes a time series in translated and scaled (dilated or compressed) versions of the mother wavelet, each one multiplied by an appropriate coefficient $[13,14]$.

Recently, wavelet analysis has been widely employed to study the non-stationary process in the time series analysis, such as the interplanetary shocks [15] and waves/ turbulence in the planetary magnetospheres [16-21]. Of special interest are the orthonormals, discrete wavelet transforms, which are used in multi-resolution analysis (MRA) $[13,22]$.

The wavelet transform is a very powerful tool to analyze the non-stationary signals, and it is used to obtain expansions of a signal using the time-localized functions (wavelets) that have good properties of localization in time and in frequency domain $[13,23]$. The wavelet transform may be continuous or discrete. While the continuous wavelet transform calculates coefficients at every possible scale, the discrete wavelet transform chooses scales and positions based on the power of the dyadic scales and positions. Thus, the discrete wavelet is a subsampling of the continuous one at just the dyadic scales $2 j-1, j=1,2, \ldots$, etc. Therefore, the discrete wavelet transform may be used in MRA, which is concerned with the study of signals or processes represented at different resolutions, and the development an efficient mechanism for going from one resolution to another [23]. 


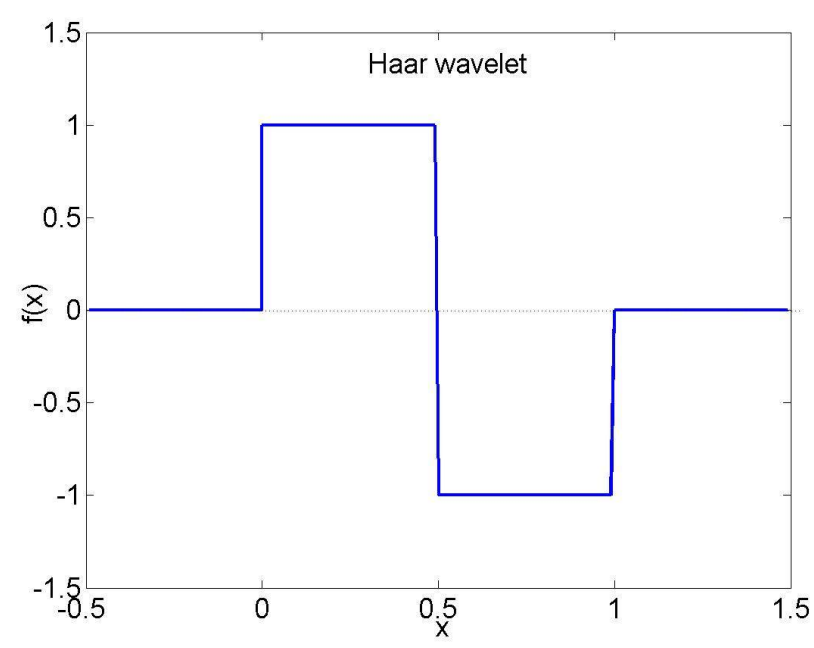

Figure 1. The Haar wavelet function.

In MRA (or multiple level decomposition), a signal (S) is broken down interactively into many lower resolution components. This decomposition process, with successive approximations being decomposed in turn, is called the wavelet decomposition tree. $S$ is split into an approximation $A$ and a detail $D$. $D$ contains the high-frequency part of the signal, whereas $A$ contains most of the characteristic frequencies in $S$. In the first step of the decomposition, $S=A 1+D 1$. In the next step, the approximation itself is split into a second level approximation: $A 1=A 2+D 2$, and so on. The process is equivalent to a filtering procedure: in the first step, the signal has a low-pass filtered component $(A 1)$, and a high-pass filtered component (D1). $A 1$ is then itself split, and one has the $D 2$ level as a band-pass filtered data. The numbers of the allowed levels depend on the length of the signal that corresponds to the dyadic order as mentioned. Thus, if a signal has 8 numbers, it is possible to decompose in three levels in order to obey the dyadic relation given by $2^{3}=8$ numbers.

\subsubsection{Haar wavelet}

In this work, the Haar wavelet is used to decompose the magnetic field vector data. Figure 1 shows a plot of this wavelet function. Since it is a discontinuous function, it should be an adequate basis to study the abrupt, discontinuous variations such as the planetary BS and MP crossings. The Haar wavelet is one of the most used orthogonal wavelet families that are used for filtering/decomposition of the time series. The Haar function is orthogonal, with dilation being dyadic in the form: $a=2^{-j}$, and its translations occur in discrete steps, in the form: $b=2^{-j} k$, where $j$ and $k$ are integers. This wavelet function is given by:

$$
\Psi_{j, k}(t)= \begin{cases}2^{j / 2} & , 2^{-j} k \leq t<2^{-j} k+1 / 2 \\ -2^{j / 2} & , 2^{-j} k \leq t<2^{-j} k+1 \\ 0 & , \text { all other values of } \mathrm{t}\end{cases}
$$

The Haar wavelet function is inserted inside the Wavelet Transform (WT) in order to decompose any non-stationary time series in dyadic scales according to equation:

$$
W T=\int f(t) \Psi_{j, k}(t) d t
$$

where $f(t)$ is the time series and $\Psi_{j, k}(t)$ is the Haar wavelet function. 


\subsection{Magnetometer data}

The high-resolution (5 s) CASSINI fluxgate magnetometer data [24], and the MESSENGER magnetometer data [25] explored in this work are obtained from NASA's Planetary Data System (PDS, http:/ / pds.jpl.nasa.gov/). This resolution was selected because it is high enough to investigate the BS and MP crossings. At the same time, these can eliminate the very high frequency noise/oscillations that can appear in the higher resolution data. The interplanetary magnetic field (IMF) measurements are performed in the radial tangential normal (RTN) coordinate system, where $\hat{\mathbf{R}}$ is directed from the Sun to the spacecraft, $\hat{\mathbf{T}}=\boldsymbol{\Omega} \times \hat{\mathbf{R}} /|\boldsymbol{\Omega} \times \hat{\mathbf{R}}|$, where $\Omega$ is the solar rotation axis, and $\hat{\mathbf{N}}$ completes the right-hand system.

\section{Results and Discussions}

\subsection{Original data}

The CASSINI spacecraft reached the near-Saturn environment during the end of June 2004 and made its first crossing of the planetary orbit [24,26]. Saturn's magnetosphere is characterized by large variations in its BS and MP boundaries due the internal factors and the solar wind pressure [9]. According to Dougherty et al. [26], were measured a total of 17 bow shock and 7 magnetopause crossings on the inbound and outbound passages. But it is important to note that the bow shock crossings were identified by abrupt increases in the magnetic field magnitude where the solar wind was compressed and decelerated. So, identification of these physical boundaries in the automatically way is important to study and understand the internal factors like the planetary periodic oscillations.

Figure 2 shows the magnetic field components measured by the CASSINI magnetometer during its inbound trajectory between 27 and 29 June 2004. The BS and MP crossings are indicated by the red and green lines, respectively. The Saturn magnetosphere size was observed to be highly variable during this period, with 7 BS and 3 MP crossings [26,27]. The BS crossings can be identified by the abrupt jumps in the magnetic field magnitude $B$. The region downstream of the BS exhibits large fluctuations in magnetic field components, indicating the magnetosheath. The MP crossings are identified by an enhancement in $B$ and by abrupt directional changes in the magnetic field components. Most of the BSs crossed during this period was found to be of the quasi-perpendicular type [27]. The BS and MP subsolar distances were found be $\sim 40-49$ Saturn radii $\left(R_{S}\right)$ and $\sim 30-34 R_{S}$, respectively [26].

The MESSENGER spacecraft made the first of its three flybys of Mercury on 14 January 2008 [20,28], and measured the near-Mercury magnetic field [25]. This is shown in Figure 3. MESSENGER, in its inbound trajectory, detected a BS, which was crossed at 18:08:38 UT (inbound) and 19:18:55 UT (outbound) on 14 January. Before the inbound MP crossing at 18:43:02, the last extended interval of the southward magnetic field ended at 18:38:40. After MESSENGER exit the magnetosphere, the magnetic field was observed to be generally northward.

\subsection{Decomposed data}

The MESSENGER magnetic field magnitude $B$ and vector components $B r, B t, B n$ during the $\sim 1 \mathrm{~h}$ Mercury flyby were decomposed in orthonormal frequency levels using the Haar wavelet transform. The magnetic field data were decomposed in 14 scales according to data length used. We performed the variance by scale in order to identify the scale with the maximum energy. Figure 4 shows the variance by scale for the wavelet coefficients $A$ and $D$ (CAs and $C D$ s) of $B$ and components $B r, B t, B n$. It can be clearly seen that most of the energy for the wavelet coefficients ( $C A s$ and $C D s$ ) is concentrated at large scales, between the scales 11 and 14 .

As the maximum energy is found to be concentrated between the scales 11 and 14, $C A$ s and $C D$ s for the scales from 1 to 10 are assigned the value 0 . This implies that the scales 1-10 do not contribute significantly to the total energy. However, the coefficients 

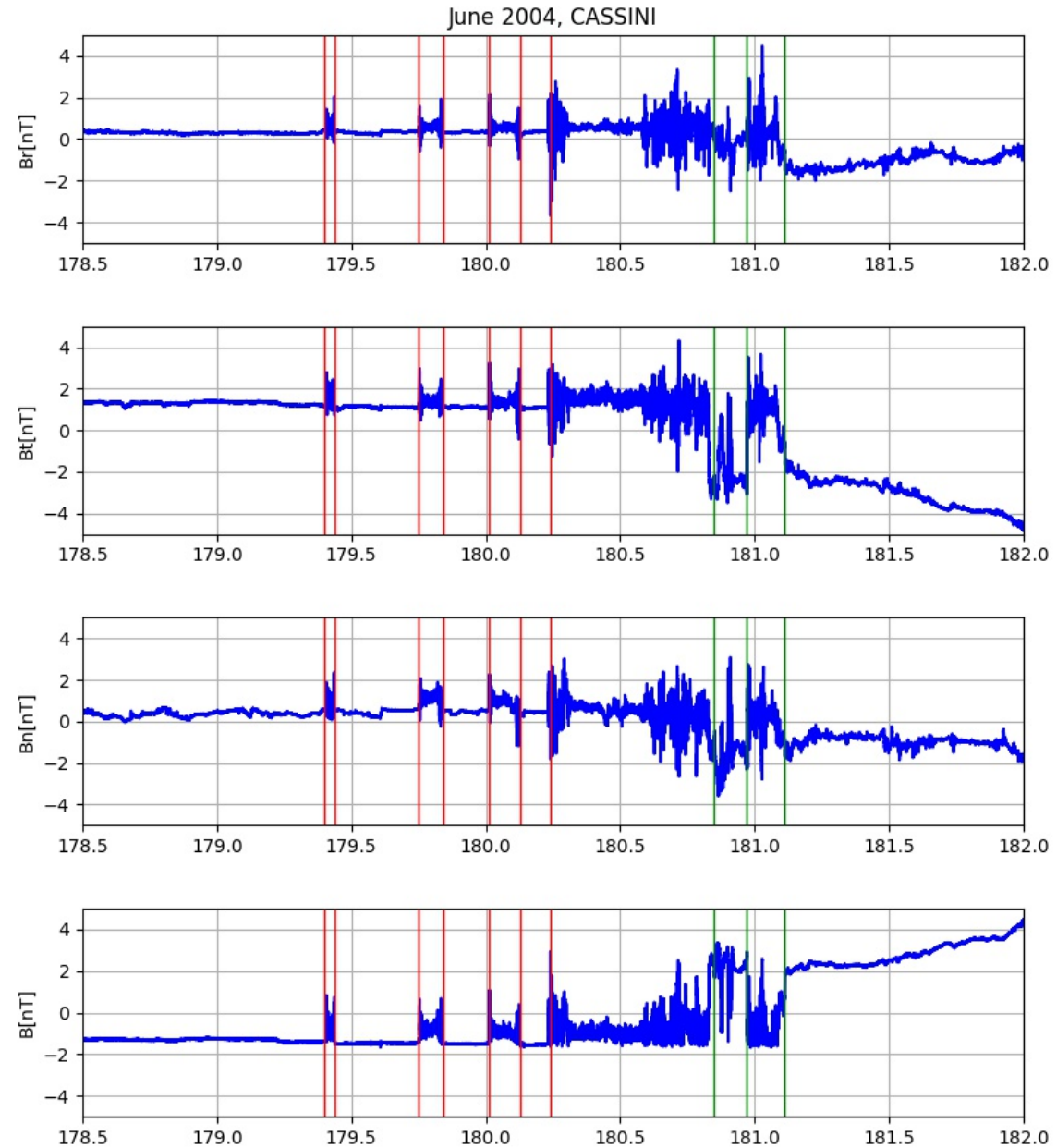

Figure 2. Near-Saturn magnetic field measurements by the CASSINI spacecraft during its inbound trajectory between 27 and 29 June 2004. The BS and MP crossings are indicated by the red and green vertical lines, respectively. 

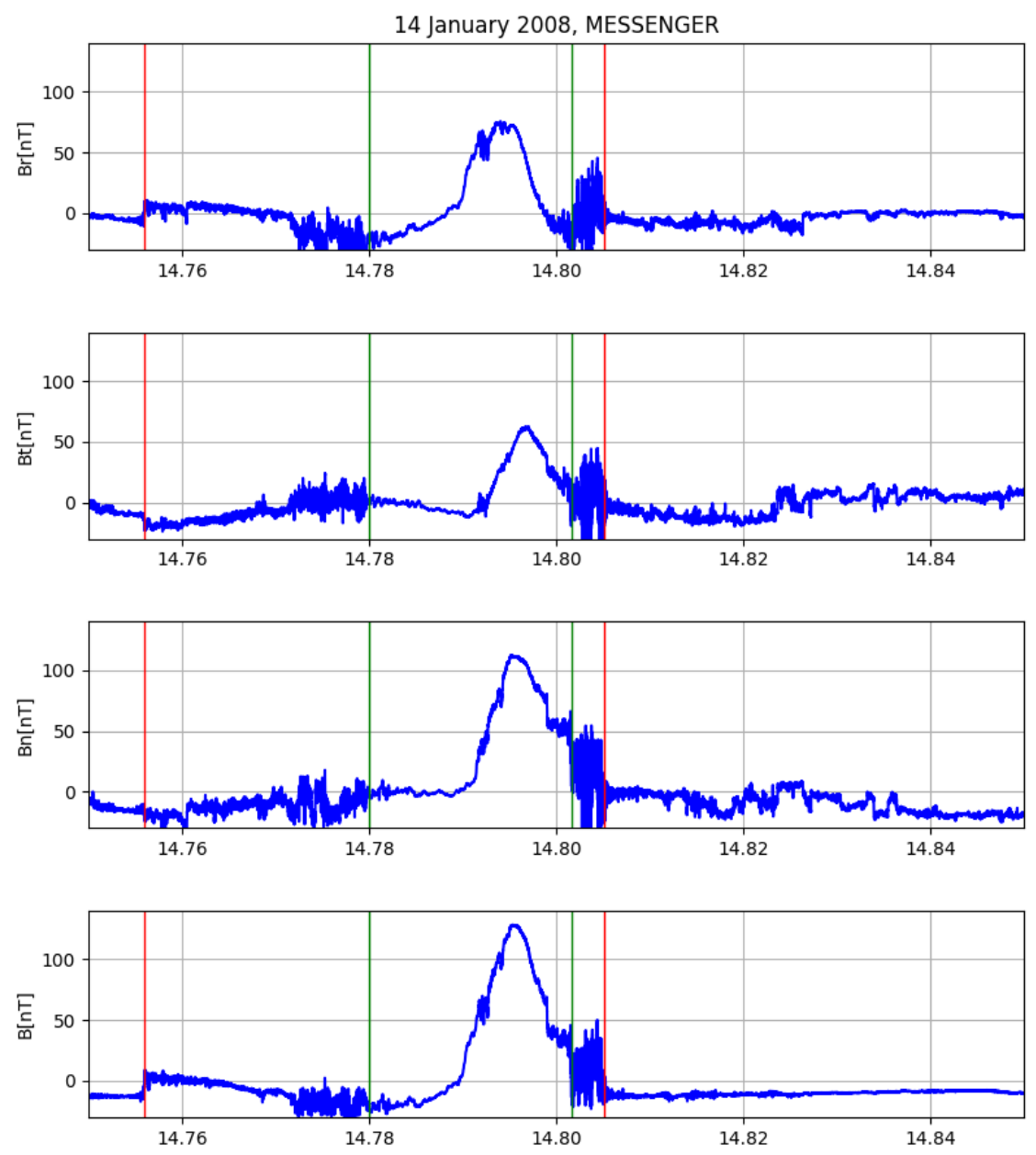

Figure 3. Near-Mercury magnetic field measurements by the MESSENGER spacecraft during its first Mercury flyby on 14 January 2008. The BS and MP crossings are indicated by the red and green vertical lines, respectively. 

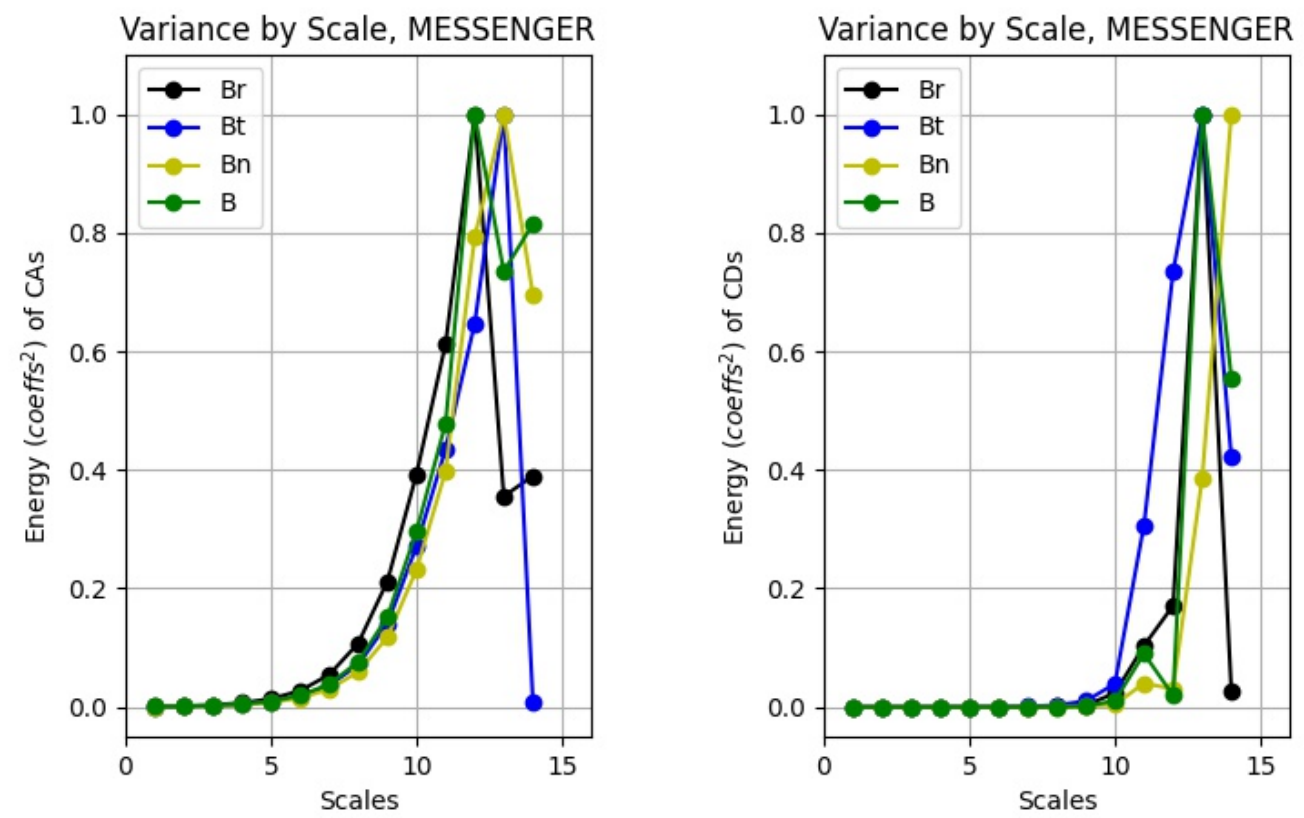

Figure 4. Variance by scale for the magnetic field measurements by MESSENGER during its Mercury flyby shown in Figure 3. Left and right panels show the variances of $C A$ s and CDs, respectively.

$C A$ s and $C D$ s were maintained from scale 11 onward. The magnetic field time series is reconstructed accordingly using the $C A$ s and $C D$ s for scales $11-14$. Figure 5 shows the reconstructed magnetic field (in red) along with the original measurements (blue). The sharp changes in the magnetic field corresponding to the BS and MP crossings can be clearly identified in the reconstructed data using the coefficients $C A s$ and $C D$ s from scale 11 to 14 .

We applied the same procedure, as above, to the CASSINI magnetic field data (for Saturn encounter). Due the greater length of the CASSINI data (compared with MESSENGER data), we decomposed it in 15 scales. Figure 6 shows the variance by scale of the $C A$ s and $C D$ s coefficients (of the three magnetic field components and magnitude). The magnetic field variances increase from scale 11 and attain the maximum at scale 15 for both wavelet coefficients. This implies that most of the energy is concentrated at large scales, from 11 to 15.

Again, we used the same methodology (as applied to the MESSENGER data), by assigning zero to the $C A$ s and $C D$ s for scales $1-10$, and those for scales $11-15$ are maintained. The magnetic field time series is reconstructed accordingly using the $C A s$ and $C D$ s of scales 11-15. Figure 7 compares the original and reconstructed magnetic fields. Clearly, the magnetic field reconstructed using only scales 11 to 15 is able identify the majority of the sharp changes associated with BS and MP crossings.

Figures 8 and 9 show the MRA decomposition levels only for the magnetic field magnitude $B$ from CASSINI and MESSENGER, respectively. The same for magnetic field components is not shown for sake of brevity and to avoid repetition. The top panels correspond to the original data, followed by different decomposition levels in the lower panels and the reconstructed data in the bottom panels. A comparison between the top and bottom panels confirms the identical shape (variation) of the original and reconstructed data. 

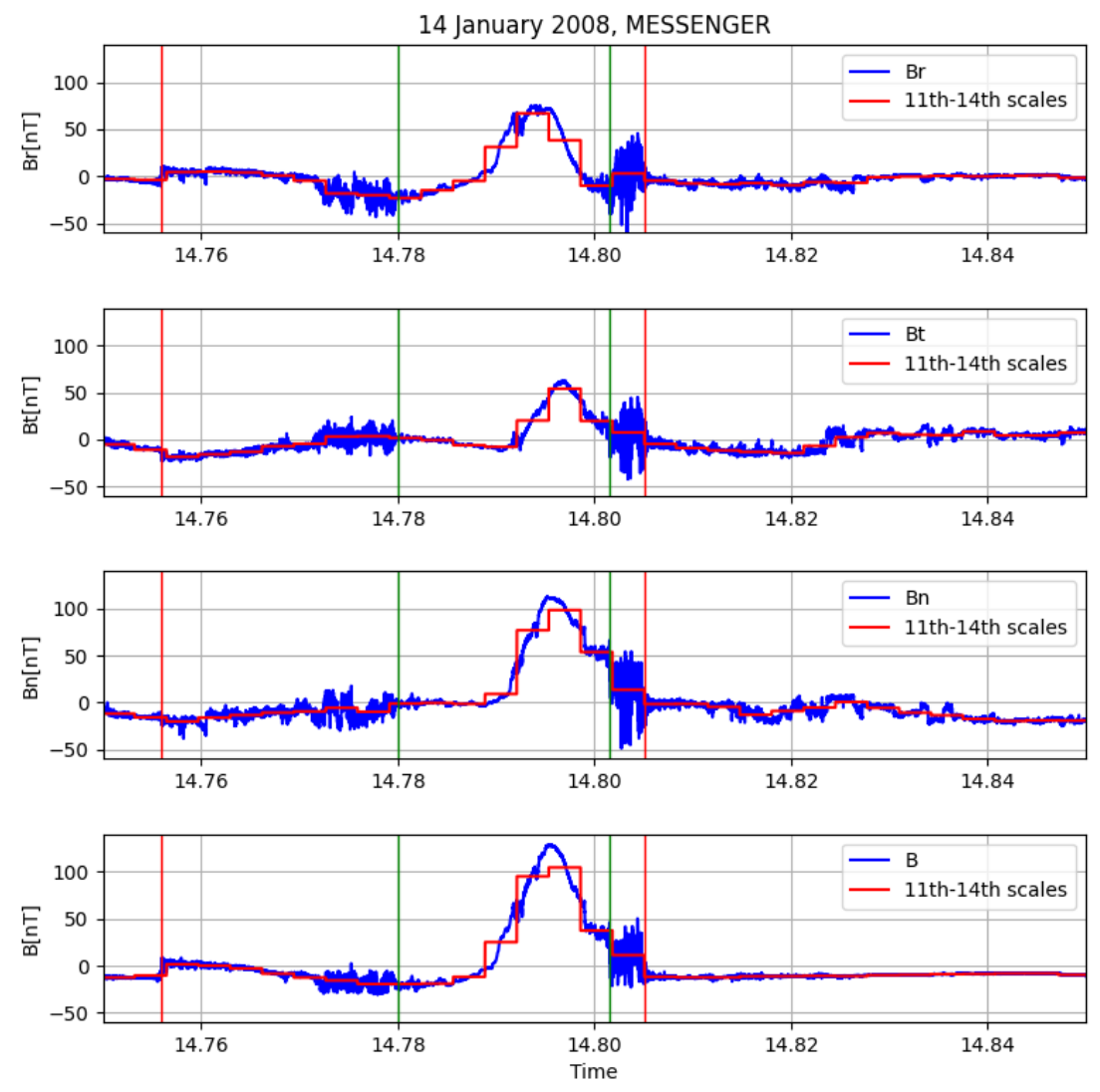

Figure 5. Comparison between the reconstructed magnetic field (red) with the MESSENGER measurements (blue) shown in Figure 3. The BS and MP crossings are indicated by the red and green vertical lines, respectively.
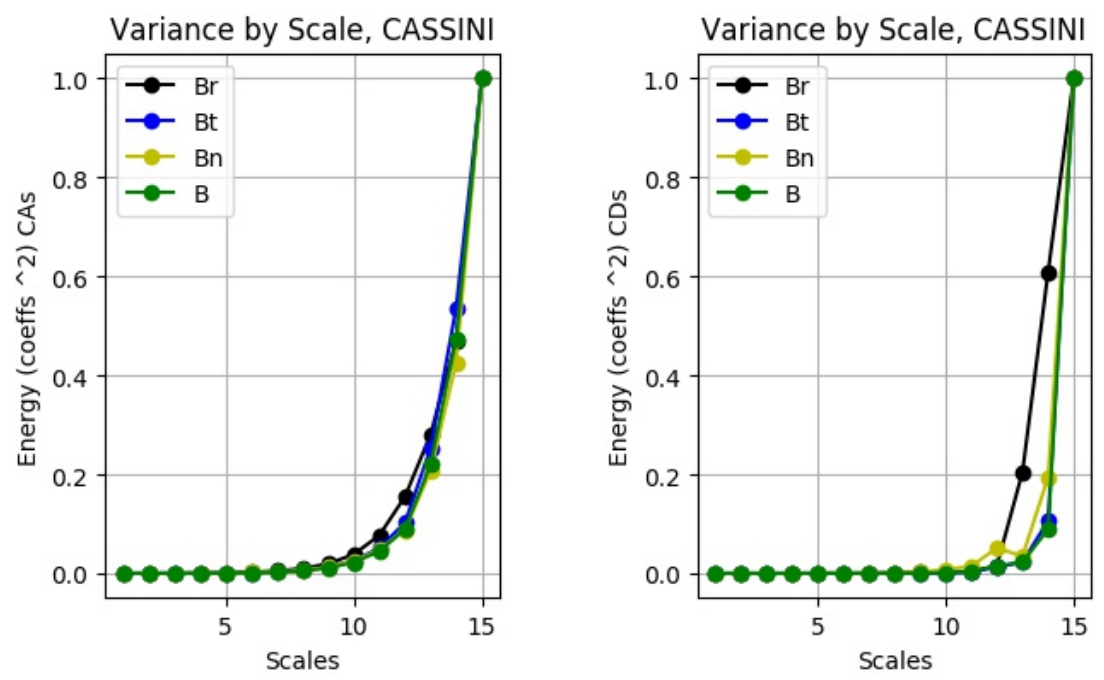

Figure 6. Variance by scale for the magnetic field measurements by CASSINI during its first Saturn flyby shown in Figure 2. Left and right panels show the variances of $C A$ s and $C D$ s, respectively. 

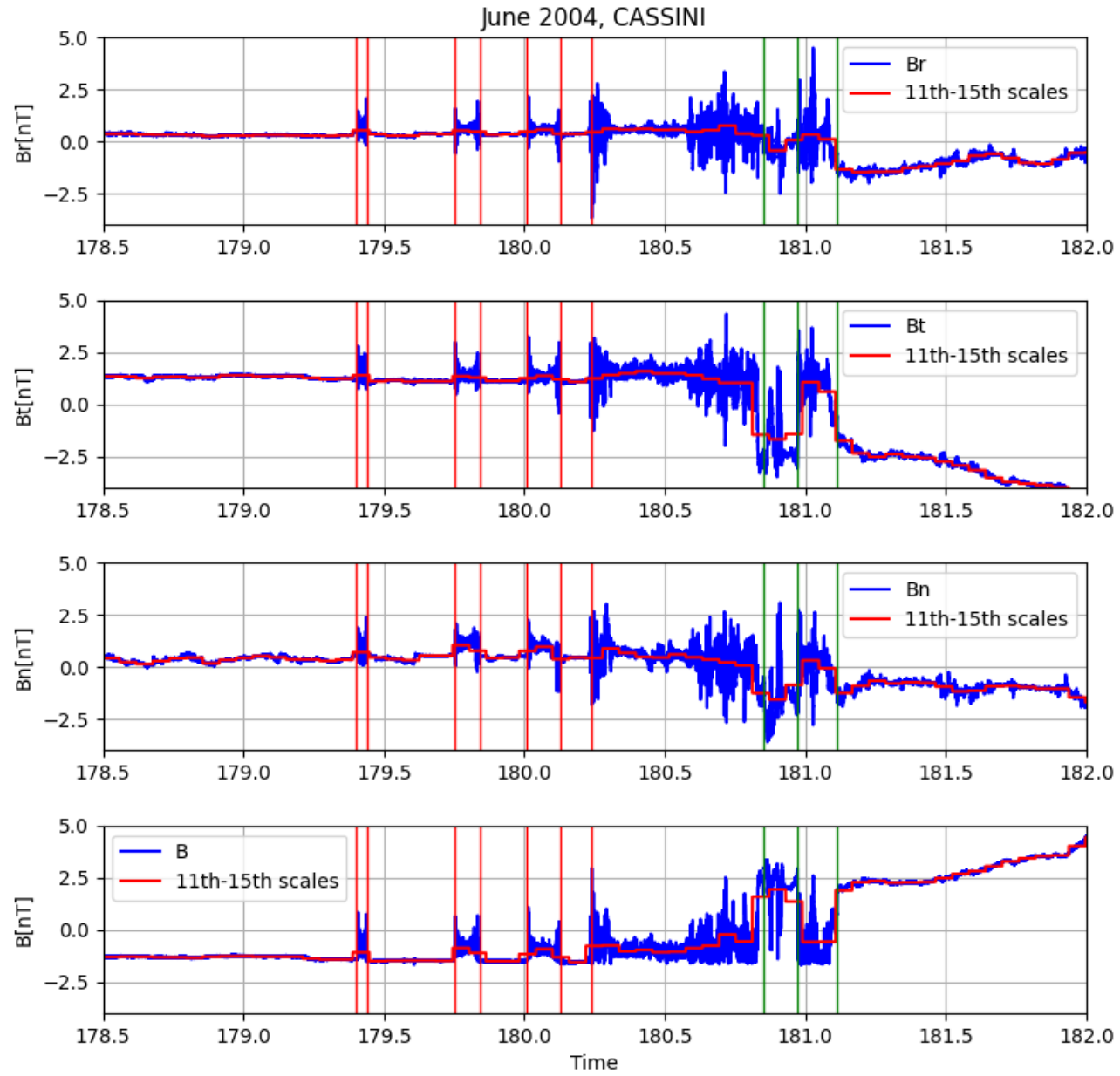

Figure 7. Comparison between the reconstructed magnetic field (red) with the CASSINI measurements (blue) shown in Figure 2. The BS and MP crossings are indicated by the red and green vertical lines, respectively. 
|B|, CASSIN|
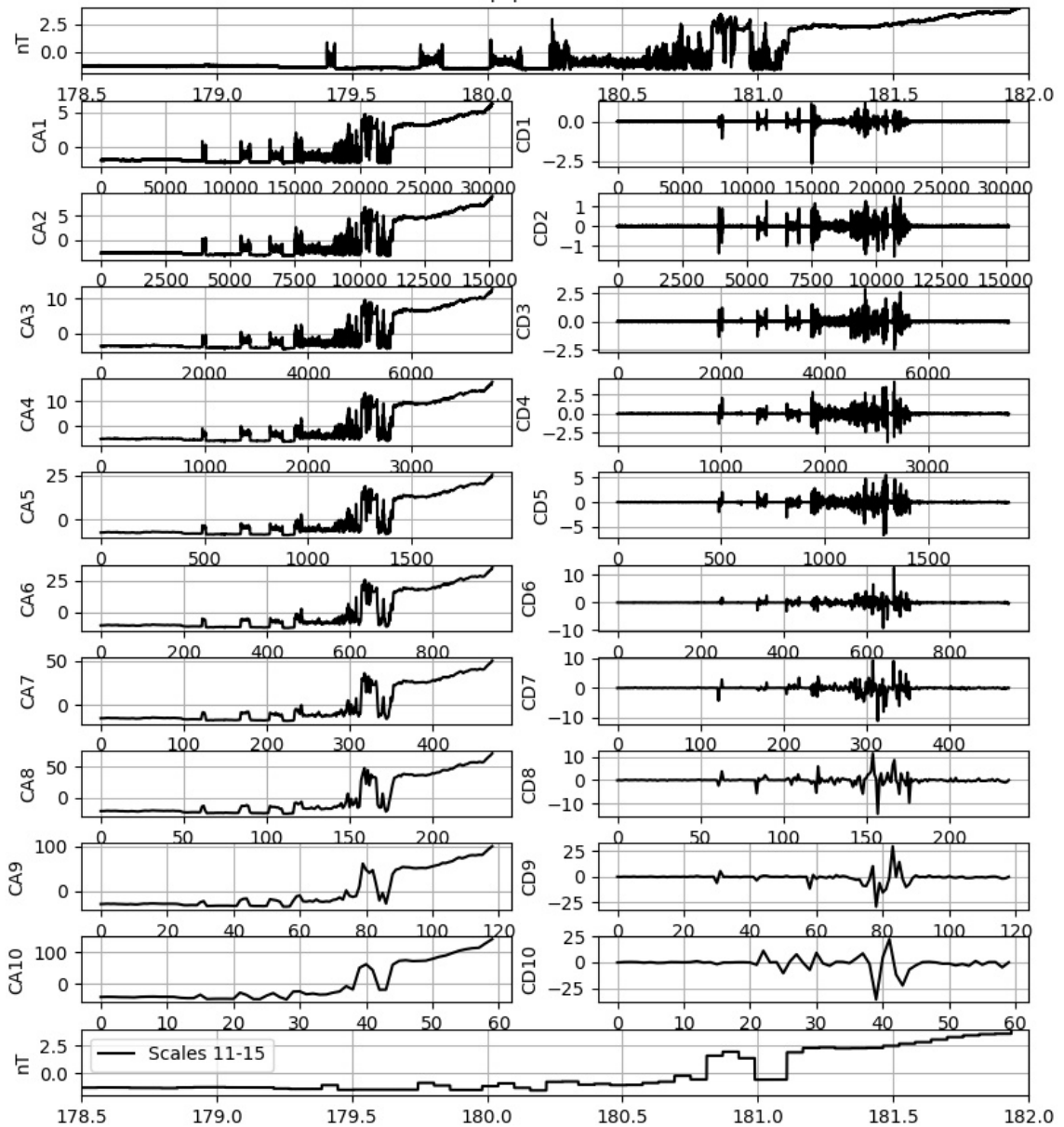

Figure 8. The Haar wavelet decomposition levels for the CASSINI magnetic field magnitude. Top panel shows the original measurement, followed by the MRA decomposition coefficients $C A 1$ to $C A 10$ (left panels), and $C D 1$ to $C D 10$ (right panels), and the reconstructed data in the bottom panel. 
$|\mathrm{B}|$, MESSENGER
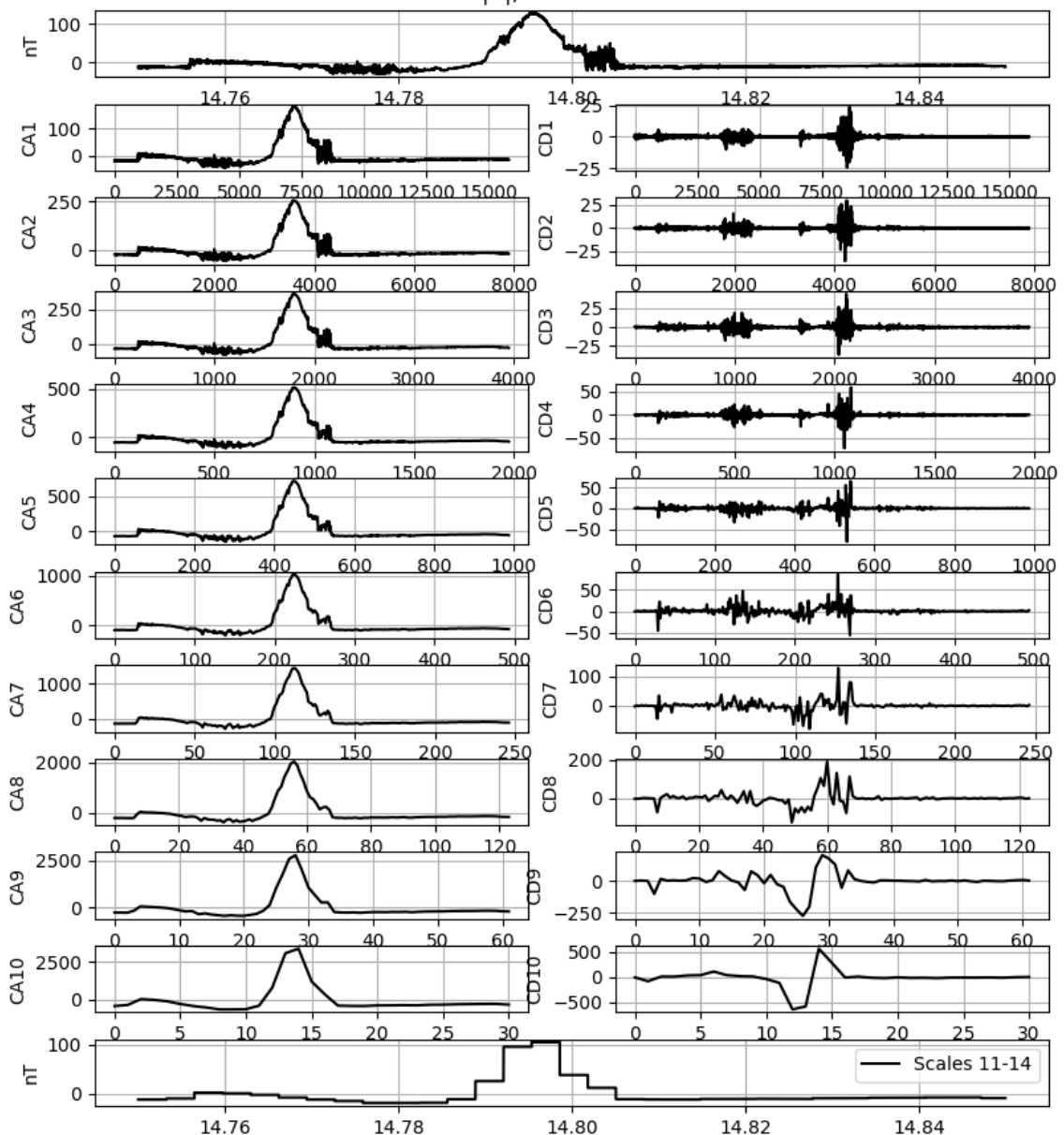

Figure 9. The Haar wavelet decomposition levels for the MESSENGER magnetic field magnitude. The panels are in the same format as in Figure 8. 


\section{Discussion and Conclusions}

In this work we presented a wavelet technique to identify the abrupt changes in the magnetic field at the magnetospheric boundary crossings. The Haar wavelet was used to decompose the magnetic field data in different frequency levels. We analyzed the magnetic field measurements by CASSINI spacecraft during its Saturn orbit insertion, and by MESSENGER during its first Mercury flyby in 2008. The Haar wavelet multiresolution analysis (MRA) was employed in this work to decompose the CASSINI and MESSENGER magnetometer data in orthonormal frequency levels. The objective was to identify the abrupt changes in the magnetic field when CASSINI and MESSENGER crossed the magnetospheric BS and MP of Saturn and Mercury, respectively. We have found that the Haar transform can be used to identify this type of abrupt boundaries.

The methodology consisted in to perform the variance by scales in order to find out the scale where the energy is maximum. After that, the CAs and CDs from 1 to 10 are assigned the value 0 for both spacecrafts. This implies that the scales $1-10$ do not contribute significantly to the total energy. Results from the variance analysis by scale it was possible to note that the maximum energy were found different scales for each spacecraft, 10 up to 11 scales $(13 \mathrm{~h}$ ) for MESSENGER and the 15 scale ( $10 \mathrm{~h}$ ) for CASSINI. Thus, although CASSINI and MESSENGER have shown maximum energy in different scales, but as the BS MPB are abrupt or quasi abrupt changes, the timescales to detect them are similar.

The BS crossings have been identified from time-scales of $\sim 10-20 \mathrm{~s}$ to $\sim 10-40 \mathrm{~min}$. However, time-scales of $\sim 10-40 \mathrm{~min}$ are more adequate to identify the BS crossings because much of the magnetic oscillations due to the magnetosheath waves are removed in these levels. On the other hand, multiple MP crossings are seen clearly only at $~ 3-$ $10 \mathrm{~h}$ time-scales. Further work should be conducted to use this technique to obtain quantitative information about the magnetospheric boundaries. This technique can be applied both to planetary boundaries as well as to detect discontinuities in the interplanetary space.

Author Contributions: Conceptualization, M.J.A.B.; methodology, M.J.A.B.; validation, M.J.A.B.; formal analysis, M.J.A.B.; writing—original draft preparation, M.J.A.B.; writing—review and editing, E.E., A.M.S.F. and R.H.. All authors have read and agreed to the published version of the manuscript.

Funding: MJAB was supported by CNPq agency contract number (PQ-302330/2015-1 and PQ305692/2018-6) and FAPEG agency contract number 2012.1026.7000905. AMSF thanks to CNPq agency (project 301969/2021-3) for the support. EE would like to thank Brazilian agencies for research grants: CNPQ/PQ (302583/2015-7, 301883/2019-0) and FAPESP (2018/21657-1). The work of RH is funded by the Science and Engineering Research Board (SERB, grant no. SB/S2/RJN080/2018), a statutory body of the Department of Science and Technology (DST), Government of India through Ramanujan Fellowship.

Institutional Review Board Statement: Not applicable.

Informed Consent Statement: Not applicable.

Data Availability Statement: The CASSINI and MESSENGER magnetometer data are obtained from NASA's Planetary Data System (PDS, http://pds.jpl.nasa.gov/).

Conflicts of Interest: The authors declare no conflict of interest.

\section{Abbreviations}

The following abbreviations are used in this manuscript: 


$\begin{array}{ll}A & \text { Approximation } \\ \text { BS } & \text { Bow shock } \\ C A & \text { Coefficient } A \\ C D & \text { Coefficient } D \\ D & \text { Detail } \\ \text { IMF } & \text { Interplanetary magnetic field } \\ \text { MP } & \text { Magnetopause } \\ \text { MRA } & \text { Multi-resolution analysis } \\ \text { PDS } & \text { NASA's Planetary Data System } \\ R_{m e} & \text { Mercury raii } \\ R_{S} & \text { Saturn radii } \\ \text { RTN } & \text { Radial tangential normal coordinate system } \\ S & \text { Signal } \\ \text { UT } & \text { Universal time }\end{array}$

\section{References}

1. Kivelson, M.G. Planetary magnetospheres. In Handbook of Space Environment 2007, 103, 469492. doi:https://doi.org/10.1007/978-3-540-46315-3_19.

2. Stern, D.P.; Ness, N.F. Planetary magnetospheres. Annual Reviews Astronomy and Astrophysics 1982, 20, 139-161. doi:https://doi.org/10.1146/annurev.aa.20.090182.001035.

3. Russell, C.T. The dynamics of planetary magnetospheres. Planetary and Space Science 2001, 49, 1005-1030. doi:https://doi.org/10.1016/S0032-0633(01)00017-4.

4. Kivelson, M.; Bagenal, F. Chapter 7. Planetary Magnetospheres. AAS/Division for Extreme Solar Systems Abstracts 2007, pp. 519-540. doi:10.1016/B978-012088589-3/50032-3.

5. Slavin, J. Mercury's magnetosphere. Advances in Space Research 2004, 33, 1859-1874. doi: 10.1016/j.asr.2003.02.019.

6. Slavin, J.; Baker, D.; Gershman, D.; Ho, G.; Imber, S.; Krimigis, S., Mercury's Dynamic Magnetosphere; 2020; p. 461-496.

7. André, N.; Dougherty, M.K.; Russell, C.T.; Leisner, J.S.; Khurana, K.K. Dynamics of the Saturnian inner magnetosphere: First inferences from the Cassini magnetometers about small-scale plasma transport in the magnetosphere. Geophysical Research Letters 2005, 32, [https://agupubs.onlinelibrary.wiley.com/doi/pdf/10.1029/2005GL022643]. doi: https://doi.org/10.1029/2005GL022643.

8. Southwood, D.J.; Chané, E. High-latitude circulation in giant planet magnetospheres. Journal of Geophysical Research: Space Physics 2016, 121, 5394-5403, [https: / agupubs.onlinelibrary.wiley.com/d doi:https://doi.org/10.1002/2015JA022310.

9. Jackman, C.M.; Thomsen, M.F.; Dougherty, M.K. Survey of Saturn's Magnetopause and Bow Shock Positions Over the Entire Cassini Mission: Boundary Statistical Properties and Exploration of Associated Upstream Conditions. Journal of Geophysical Research - Space Physics 2019, 124, 85-89. doi:https: / / doi.org/10.1029/2019JA026628.

10. Waite, J.H.; Combi, M.R.; Ip, W.H.; Cravens, T.E.; McNutt, R.L.; Kasprzak, W.; Yelle, R.; Luhmann, J.; Niemann, H.; Gell, D.; Magee, B.; Fletcher, G.; Lunine, J.; Tseng, W.L. Cassini Ion and Neutral Mass Spectrometer: Enceladus Plume Composition and Structure. Science 2006, 311, 1419-1422, [https://science.sciencemag.org/content/311/5766/1419.full.pdf]. doi:10.1126/science.1121290.

11. Bolzan, M.J.A.; Franco, A.M.S.; Echer, E. A wavelet based method to remove the long term periodicities of geophysical time series. Advances in Space Research 2020, 66, 299-306. doi: https://doi.org/10.1016/j.asr.2020.04.014.

12. Bolzan, M.J.A.; Guarnieri, F.L.; Vieira, P.C. Comparisons between two wavelet functions in extracting coherent structures from solar wind time series. Brazilian Journal of Physics 2009, 39, 12-17. doi:https://doi.org/10.1590/S0103-97332009000100002.

13. Kumar.; Foufoula-Georgiou. Wavelet analysis for geophysical applications. Reviews of Geophysics 1997, 35, 385-412. doi:https://doi.org/.

14. Torrence, C.; Compo, G.P. A Practical Guide to Wavelet Analysis. Bulletin of the American Meteorological Society 1998, 79, 61-78. doi:https://doi.org/10.1175/1520 0477(1998)079<0061:APGTWA>2.0.CO;2.

15. Gedalin, M.; Newbury, J.A.; Russell, C.T. Shock profile analysis using wavelet transform. Journal of Geophysical Research 1998, 103, 6503-6511. doi:https:/ / doi.org/. 
16. Tarasov, V.; Dubinin, E.; Perraut, S.; Roux, A.; Sauer, K.; Skalsky, A.; Delva, M. Wavelet application to the magnetic field turbulence in the upstream region of the Martian bow shock. Earth, Planets and Space 1998, 50, 699-708. doi:https:/ / doi.org/10.1186/BF03352163.

17. Espley, J.R.; Cloutier, P.A.; Brain, D.A.; Crider, D.H.; Acuna, M.H. Observations of lowfrequency magnetic oscillations in the margina magnetosheath, magnetic pileup region and tail. Journal of Geophysical Research 2004, 109. doi:https:/ / doi.org/10.1029/2003JA010193.

18. Bolzan, M.J.A.; Sahai, Y.; Fagundes, P.R.; Rosa, R.R.; Ramos, F.M.; Abalde, J.R. Intermittency analysis of geomagnetic storm time-series observed in Brazil. Journal of Atmospheric and Solar-Terrestrial Physics 2005, 67, 1365-1372. doi:https:/ / doi.org/10.1016/j.jastp.2005.06.008.

19. Echer, E. Foreshock and magnetosheath waves at Uranus and Neptune studied with wavelet analysis. Advances in Space Research 2009, 44, 1030-1037. doi:https://doi.org/10.1016/j.asr.2009.05.02

20. Echer, E. Wavelet analysis of ULF waves in the Mercury's magnetosphere. Revista Brasileira de Geofísica 2010, 28, 175-182. doi:https:/ / doi.org/10.1590/S0102-261X2010000200003.

21. Franco, A.M.S.; Franz, M.; Echer, E.; Bolzan, M.J.A. Wavelet analysis of low frequency plasma oscillations in the magnetosheath of Mars. Advances in Space Research 2020, 65, 2090-2098. doi:https://doi.org/10.1016/j.asr.2019.09.009.

22. Echer, E. Multi-resolution analysis of global total ozone column during 1979-1992 Nimbus-7 TOMS period. Annales Geophysicae 2004, 22, 1487-1493. doi:https:/ / doi.org/10.5194/angeo22-1487-2004.

23. Percival, D.B.; Walden, A.T. Wavelet Methods for Time Series Analysis; Cambridge University Press, 2000.

24. Dougherty, M.K.; Kellock, S.; Southwood, D.J.; Balogh, A.; Smith, E.J.; Tsurutani, B.T.; Gerlach, B.; Glassmeier, K.H.; Gleim, F.; Russell, C.T.; Erdos, G.; Neubauer, F.M.; Cowley, S.W.H. The Cassini magnetic field investigation. Space Science Reviews 2004, 114, 331-338. doi:https://doi.org/10.1007/s11214-004-1432-2.

25. Anderson, B.J.; Acuña, M.H.; Lohr, D.A.; Scheifele, J.; Raval, A.; Korth, H.; Slavin, J.A. The Magnetometer Instrument on MESSENGER. Space Science Reviews 2007, 131, 417—450. doi: https:/ / doi.org/10.1007/s11214-007-9246-7.

26. Dougherty, M. K. Achilleos, N.; Andre, N.; Arridge, C.S.; Balogh, A.; Bertucci, C.; Burton, M.E.; Cowley, S.W.H.; Erdos, G.; Giampieri, G.; Glassmeier, K.H.; Khurana, K.K.; Leisner, J.; Neubauer, F.M.; Russell, C.T.; Smith, E.J.; Southwood, D.J.; Tsurutani, B.T. Cassini magnetometer observations during Saturn orbit insertion. Science 2005, 307, 1266-1270. doi: https:/ / doi.org/10.1126/science.1106098.

27. Achilleos, N.e.a. Orientation, location and velocity of Saturn's bow shock: Initial results from the Cassini spacecraft. Journal of Geophysical Research 2006, 111, xx-xx. doi: https:/ / doi.org/10.1029/2005JA011297.

28. Slavin, J.A.; Acuña, M.H.; Anderson, B.J.; Baker, D.N.; Benna, M.; Gloeckler, G.; Gold, R.E.; Ho, G.C.; Killen, R.M.; Korth, H.; Krimigis, S.M.; McNutt Jr., R.L.; Nittler, L.R.; Raines, J.M.; Schriver, D.; Solomon, S.C.; Starr, R.D.; Trávníček, P.; Zurbuchen, T.H. Mercury's magnetosphere after MESSENGER's first flyby. Science 2008, 321, 85-89. doi: https:/ /doi.org/10.1126/science.1159040. 